\title{
3D Prostate Segmentation in Ultrasound Images Based on Tapered and Deformed Ellipsoids
}

\author{
Seyedeh Sara Mahdavi ${ }^{1}$, William J. Morris ${ }^{2}$, Ingrid Spadinger ${ }^{2}$, Nick Chng ${ }^{2}$, \\ Orcun Goksel ${ }^{1}$, and Septimiu E. Salcudean ${ }^{1}$ \\ ${ }^{1}$ Department of Electrical and Computer Engineering, University of British \\ Columbia, Vancouver, Canada \\ \{saram, orcung, tims\}@ece.ubc.ca \\ 2 Vancouver Cancer Center, British Columbia Cancer Agency, Vancouver, Canada \\ \{jmorris, ispading, nchng\}@bccancer.bc.ca
}

\begin{abstract}
Prostate segmentation from trans-rectal transverse B-mode ultrasound images is required for radiation treatment of prostate cancer. Manual segmentation is a time-consuming task, the results of which are dependent on image quality and physicians' experience. This paper introduces a semi-automatic 3D method based on super-ellipsoidal shapes. It produces a 3D segmentation in less than 15 seconds using a warped, tapered ellipsoid fit to the prostate. A study of patient images shows good performance and repeatability. This method is currently in clinical use at the Vancouver Cancer Center where it has become the standard segmentation procedure for low dose-rate brachytherapy treatment.
\end{abstract}

\section{Introduction}

Low dose rate (LDR) prostate brachytherapy is a common radiation treatment for early stage prostate cancer. It consists of the permanent implant of 40-100 small radioactive seeds into the prostate with the aim of delivering a sufficiently high radiation dose to the cancerous tissue, while maintaining a tolerable dose to the urethra and rectum. Possible side effects of this procedure include urinary incontinence and erectile dysfunction which are mainly related to inaccurate delivery of the seeds caused by inaccurate visualization of the prostate preoperatively and intra-operatively.

In a typical pre-operative trans-rectal volume study, a series of 9-14 parallel trans-rectal ultrasound (TRUS) images, from the base to the apex, are collected. These images are then manually segmented to create a 3D model of the prostate, which is used to generate a treatment plan. In typical TRUS images, the prostate is the largest and its boundary is the most discernible in the mid-gland section. Approaching the base and the apex, the boundary almost disappears into the background. Segmentation of these two regions is mainly based on experience and by looking at the mid-portion of the gland, which gives a hint of how far the prostate extends axially. Manual segmentation often requires 5-10 minutes and, in addition to image quality, it greatly relies upon the experience and peculiar habits of the clinician. Therefore, a suitable (semi-)automatic segmentation algorithm can result in less variable contours generated in less time. 
Many (semi-)automatic prostate segmentation methods on ultrasound images have been proposed during the last few years [1. Some methods are based on image enhancement or edge detection techniques such as 234. However, methods which rely solely on image information are sensitive to image quality and noise level. Additional information about the shape of the prostate can increase robustness to noise and reduce segmentation time. Deformable models have been widely used for medical image segmentation [5,6/7,8,9] and are generally more successful than the former methods. Fitting ellipses, ellipsoids, super-ellipses, and deformable ellipses or using them for initialization have been relatively attractive approaches for prostate segmentation due to the shape of the gland 1011121314 .

To the best of our knowledge, there have been no other reports of a 3D (semi) automatic prostate segmentation method reliable enough and fast enough for effective replacement of standard manual segmentation in clinical use. While the extensive study of 12 showed good segmentation results, the method presented is limited to 2D. Previous 3D methods [1516] are time consuming ( $>2$ minutes) or require significant user intervention [11. Our use of an a priori shape allows prostate segmentation in poorly visible regions and therefore can be used in standard clinical practice, thus enabling a more complete validation study than presented with other 3D methods.

In this paper, we propose a method that generates the $3 \mathrm{D}$ volume of the prostate based on a combination of the physician's experience, edge detection and prior knowledge of the prostate's shape. The method introduced in [14 has been extended to account for the tapered 3D shape of the prostate. Furthermore, we introduce a novel validation method based on different regions of the gland. This method enables a critical evaluation of prostate segmentation algorithms based on their importance in treatment planning.

\section{Methods}

\subsection{Algorithm}

Based on the prostate's shape and considering the effect of the TRUS probe pressure on the gland, we found a warped and tapered ellipsoid with tapering in three axes suitable as the final 3D volume of the prostate. This can be constructed from 2D warped and tapered ellipses generated from each image slice. However, fitting such shapes for each slice can be time-consuming. A solution to this problem is to un-warp and un-taper all images in a pre-processing step that makes the prostate shape approximately ellipsoidal. Un-warping is carried out to remove the posterior concavity of the prostate formed due to TRUS pressure on the gland. Transversal un-tapering is then applied leaving a simple convex ellipse fitting problem for each image. Finally an axially tapered ellipsoid or an 'egg shape' with an elliptical cross-section is fitted to the $2 \mathrm{D}$ contours and the results are inversely warped and tapered to match the actual prostate shape. Details of the algorithm are as follows. 

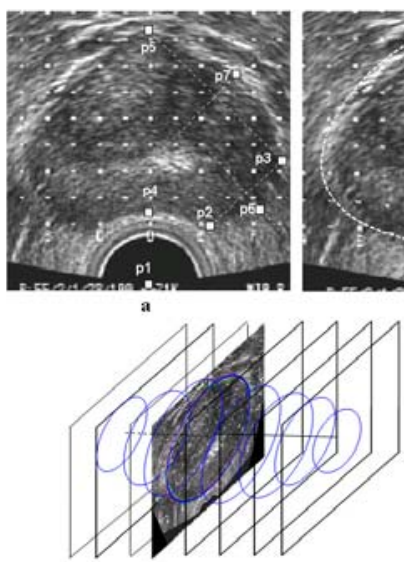

d

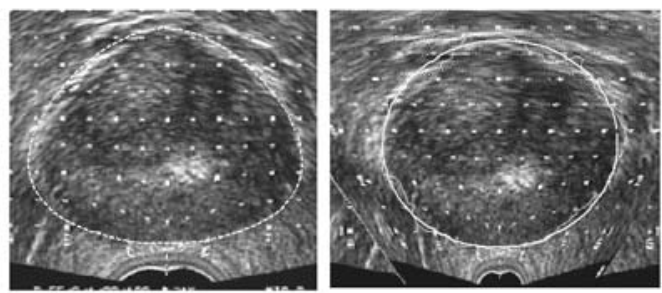

c

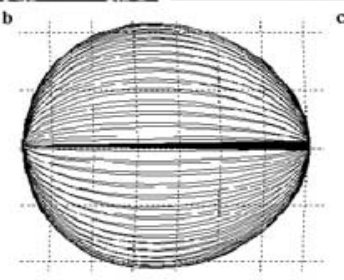

e

Fig. 1. (a) Initial points, (b) Un-warped mid-gland with initial tapered ellipse fit, (c) Un-tapered, un-warped image with edge detection and ellipse fit, (d) propagation from mid-gland to the remaining slices, (e) tapered ellipsoid

Initialization: The segmentation algorithm is initialized with the user selecting the mid-gland, apex, and base slices. The base and apex are the extreme slices in which the prostate can be seen, superiorly and inferiorly, and the mid-gland slice usually contains the largest and most visible section of the gland. The "slices" used for segmentation throughout the algorithm will be from base +1 to ape -1 . On the mid-gland slice, the user selects $p_{1}$ the TRUS probe center and six boundary points which are (Fig 1(a)): $p_{2}$-lowest posterior lateral, $p_{3}$-extreme right, $p_{4}$-medial posterior, $p_{5}$-medial anterior, $p_{6}\left(p_{7}\right)$ - intersection of the midperpendicular line between $p_{2}$ and $p_{3}\left(p_{3}\right.$ and $\left.p_{5}\right)$ with the boundary. The aim is to extract the most information from the image while keeping the variability of the initialization low by directing the user to specific regions (superior, lateral, inferior) and using guiding lines.

Un-warping: Based on the selected points, the mid-gland image and initial points are un-warped 13 to reduce the deformation caused by the TRUS probe, using $r_{n e w}=r-r \sin (\theta) \exp \left(-r^{2} / 2 \sigma^{2}\right)$ where $r$ is the current distance of an image pixel on a radial line starting from the probe center with angle $\theta$ ( $\theta=90^{\circ}$ being the medial line) and $r_{n e w}$ is the distance of the re-located pixel. According to this sinusoidally weighted Gaussian function, the maximum deformation is achieved when $\theta=90^{\circ}$ and is reduced as the distance to the center of the probe increases. The amount of radial stretch is determined by $\sigma$ which is calculated using the three initial points $p_{1}, p_{2}$, and $p_{4}$ for $\theta=90^{\circ}$.

Un-tapering: To the un-warped initial points, a tapered ellipse is fitted by solving the following problem using the Levenberg-Marquardt optimization method with the goal of obtaining the parameters $P=\left(x_{0}, y_{0}, a_{x}, a_{y}, t_{1}\right)$ : 


$$
\begin{gathered}
\min _{P}\left\{e^{T} e \mid e_{i}=\sqrt{a_{x} a_{y}}\left[\left(\frac{x_{i}^{\prime}}{a_{x}}\right)^{2}+\left(\frac{y_{i}^{\prime}}{a_{y}}\right)^{2}-1\right], \quad i=1 \ldots N\right\} \\
x_{i}^{\prime}=\left(x_{i}-x_{0}\right) /\left(\frac{t_{1}}{a_{y}}\left(y_{i}-y_{0}\right)+1\right), \quad y_{i}^{\prime}=\left(y_{i}-y_{0}\right)
\end{gathered}
$$

with $-1 \leq t_{1} \leq 1$ being the tapering parameter $\left(t_{1}=0\right.$ for ellipse), $\left[x_{0}, y_{0}\right]$ the center of the shape, $a_{x}$ and $a_{y}$ the radii along the $x$ and $y$ axes, and $\left[x_{i}, y_{i}\right]$ the coordinates of the $N$ boundary points on the mid-gland image. $N$ is determined by the initial points and their reflections about the mid-sagittal plane (Fig[1(b)).

IMMPDA edge detection in mid-gland: The resulting tapered ellipse is used to guide the IMMPDA edge detection algorithm [17] by setting limits on how far from the initial contour the edge detection can search. In order to improve the tapered ellipse fitting, the resulting edge points are once again fed to the LevenbergMarquardt algorithm. The tapering value of this contour, $t_{1}$, is used to 'un-taper' the ultrasound images. We assume that the prostate is most tapered at the midgland and the tapering linearly reduces to zero towards the base and the apex. Thus, only one tapering parameter is computed for the entire set of transverse prostate images. Using the negative of the tapering value for each slice, all the images along with the initial points are un-tapered. The combination of un-warping and un-tapering of the images creates images in which the prostate is approximately elliptical in shape, thus simplifying the problem to the convex problem of fitting an ellipse. An ellipse can be fitted to data points by solving a generalized eigenvector problem. Fig 1(c) shows the un-warped and un-tapered mid-gland image along with the IMMPDA edge detection result and final fitted ellipse.

Contour propagation and IMMPDA detection in remaining slices: In order to find the prostate boundary in the rest of the slices, two semi-ellipsoids are first fitted (again using the generalized eigenvector problem): one to the midgland contour and the intersection of a line passing the center of the mid-gland contour and parallel to the TRUS with slice base - 1, and the second to the mid-gland contour and the intersection of the same line with slice apex +1 . The intersection of the two semi-ellipsoids with each of the slices creates initial contours for the delineation of the remaining slices (Fig 1(d)). The IMMPDA edge detection algorithm is applied to each image and the detected edge points are used to fit the final 3D shape. Two semi-ellipsoids instead of one ellipsoid are used because the mid-gland slice is usually closer to the base, thus giving a better initial approximation for the remaining contours.

3D Tapered ellipsoid fit: A tapered ellipsoid is fitted as a final 3D shape to the slice contours obtained (Fig[1(e)). This is no longer a convex problem, and we solve it using the Levenberg-Marquardt algorithm. The following optimization problem is solved in order to find the tapered ellipsoid parameters $P=\left(x_{0}, y_{0}, z_{0}, a_{x}, a_{y}, a_{z}, t_{2}, t_{3}\right)$ :

$$
\begin{gathered}
\min _{P}\left\{e^{T} e \mid e_{i}=\sqrt{a_{x} a_{y} a_{z}}\left[\left(\frac{x_{i}^{\prime}}{a_{x}}\right)^{2}+\left(\frac{y_{i}^{\prime}}{a_{y}}\right)^{2}+\left(\frac{z_{i}^{\prime}}{a_{z}}\right)^{2}-1\right], \quad i=1 \ldots M\right\} \\
x_{i}^{\prime}=\left(x_{i}-x_{0}\right) /\left(\frac{t_{2}}{a_{z}}\left(z_{i}-z_{0}\right)+1\right), \\
y_{i}^{\prime}=\left(y_{i}-y_{0}\right) /\left(\frac{t_{3}}{a_{z}}\left(z_{i}-z_{0}\right)+1\right), z_{i}^{\prime}=\left(z_{i}-z_{0}\right)
\end{gathered}
$$




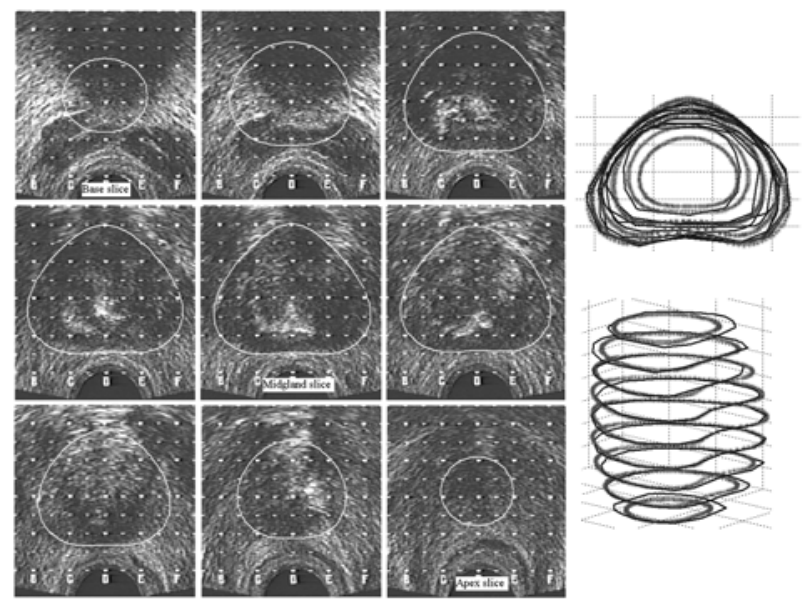

Fig. 2. Final contours on the TRUS B-mode images and the final 3D shape compared with manual segmentation (thin lines)

where $a_{x}, a_{y}, a_{z}$ are the radii along the axes, $\left[x_{0}, y_{0}, z_{0}\right]$ is the position of the center of the volume, $t_{2}$ and $t_{3}$ are respective $x$ and $y$ tapering values in the direction of the ultrasound probe axis and $\left[x_{i}, y_{i}, z_{i}\right]$ are the coordinates of $M$ boundary points segmented in the image slices.

The $3 \mathrm{D}$ volume fitting is the most time consuming part of the algorithm. To aid the optimization algorithm, an ellipsoid is initially fitted to the data cloud consisting of the ellipse contours of all slices. Since this is a convex problem, the one and only minimum is found almost instantly. The derived center of the ellipsoid and the axes are used along with the two tapering parameters, initially set to 0.05 assuming slight tapering of the prostate towards the apex, as initial values for the optimization algorithm in Eq,2.

Volume re-slicing, tapering and warping: The final volume is sliced, and the resulting contours are inversely tapered and warped. Fig 2 shows the resulting contours in the TRUS B-mode images and a comparison of the final 3D volume generated by the algorithm with the manual contours created by a physician.

\subsection{Evaluation}

The current algorithm for prostate segmentation is now been used by therapists at the Vancouver Cancer Center, where approximately 300 patients per year are treated. Before treatment planning, radiation oncologists observe and modify the resulting contours, if needed. To evaluate this algorithm we have carried out three comparison studies between 3D shapes generated from: $(i)$ pre- and post-modified semi-automatic contours, $(i i)$ original and repeated pre-modified semi-automatic contours and finally (iii) manually segmented contours and premodified semi-automatic contours. 


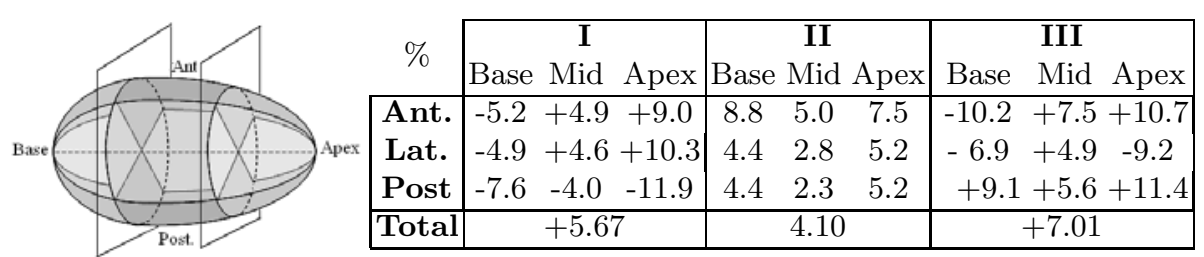

Fig. 3. Percentage of volume errors for each sector. I: pre- vs. post modified semi-auto, II: original vs. repeated semi-auto, III: semi-auto vs. manual.

In all three studies, the 3D prostate shape has been divided into nine sectors (Fig[3). Division in the transverse plane produces three anterior, posterior and lateral regions and axial division results in the apex, base and mid regions. The reason for such a division is to best represent the critical regions involved in the treatment. For example, the boundary of the mid-posterior region should be accurately identified to avoid rectal complications.

For each sector, the percentage of volume error, $V_{e r r}$, is calculated. This value is the percentage of the non-overlapping volume of two 3D shapes, as defined in comparison studies $(i),(i i)$ and $(i i i)$, to the sum of their volumes. Additionally, the percentage of total volume difference, $V_{\text {diff }}$, is also reported. This is $\left(V_{A}-\right.$ $\left.V_{B}\right) / V_{B} \times 100$, with $V_{A}$ and $V_{B}$ being the pre- and post-modified (study $(i)$ ) and pre-modified and manual volumes (study $($ iii $)$ ), respectively.

Comparison between the pre- and post-modified semi-automatic contours can give a measure of how satisfied the physicians are with the results of the algorithm. This is done on 22 sets of prostate images with an average of 10 images per case. To evaluate the repeatability of the algorithm, 10 of the 22 cases were randomly selected for repeated segmentation. The repeated contours are compared with the pre-modified contours using the same nine-sector analysis.

One can argue that seeing the semi-automatic contours may bias the physician's judgment of where the prostate boundary actually is. To determine the extent to which the a priori shapes generated by the algorithm may cause such bias, 11 cases were segmented both manually (by an expert) and by our algorithm (by a volunteer trained by an expert).

The Mean Absolute Distance (MAD), and Maximum Difference (MAXD) were also calculated for the mid-gland slices in studies $(i)$ and $(i i i)$ as measures of the $2 \mathrm{D}$ contouring ability of the algorithm. The former(latter) is the average(maximum value) of the absolute radial distance between the two contours to be compared. Finally, a B-mode/MRI volume comparison is carried out.

\section{Results}

The average normalized volume error for the nine sectors can be seen in the following tables (Fig (3). Column I shows $V_{\text {err }}$ between semi-automatic contours and their modified versions for the 22 cases. The positive(negative) signs indicate that the sector is smaller (larger) in the modified version. $V_{\text {diff }}$ is $0.61 \pm 7.7(\%)$ 
and the average MAD/MAXD for the mid-gland slice is $0.66 \pm 0.5 / 2.06 \pm 1.59$ $(\mathrm{mm})$ in which 4 of the 22 cases did not need any modification in the midgland slice. In order to study the repeatability of the algorithm, 10 of these cases were randomly selected and segmented by a different user two weeks after initial segmentation, the results of which are seen in column II. Finally, $V_{\text {err }}$ between semi-automatic contours and manual contours of the 11 cases is shown in column III. In this case, a positive(negative) sign means that the manual sector is smaller (larger). The percentage volume errors are mainly of the order of the pre- and post-modified comparison, which implies that the physician is not significantly biased by the semi-auto contours. $V_{\text {diff }}$ is $1.8 \pm 5.1(\%)$ and the MAD/MAXD for the mid-gland slice is $1.25 \pm 0.38 / 3.17 \pm 0.73(\mathrm{~mm})$. Comparison of pre- and post-modified semi-automatic contours with manual MRI contours of 8 patient prostates showed a $V_{\text {diff }}$ of $13.1 \pm 7.5(\%)$ between pre-modified and MRI contours. Interestingly, this value is $8.6 \pm 5.3(\%)$ for post-modified and MRI contours (in both cases the MRI volume usually being smaller). This suggests that even the modified contours used for treatment planning create a larger volume than that seen in MRI.

The duration from the moment the initial points are selected until the $3 \mathrm{D}$ shape is generated is $14.36 \pm 1.39 \mathrm{~s}(\max .17 \mathrm{~s})$ on a standard PC.

\section{Conclusion and Discussion}

We have presented a fast algorithm for semi-automatic segmentation of the prostate. The algorithm is presently in clinical use (to date plans for 90 patients were generated) and provides an initial segmentation to radiation oncologists performing brachytherapy. It was shown that only small modifications are required to the initial segmentations, specifically in the mid region of the gland.

It is important to note that the physicians definition of segmentation may not necessarily be what the actual boundaries of the prostate are in B-mode images. Contouring is also affected by how the treatment is planned and carried out. This can be seen in the comparison with MR images which are well known for providing better visibility of the gland. Our results showed that even segmentation results of B-mode images approved for planning have larger volumes than those created from MRI.

A major advantage of this algorithm is that it produces smooth and symmetric contours, in a manner that is physician independent and repeatable. These are suitable for treatment planning and desired by physicians. The algorithm is fast enough to be used in pre-planning. It requires some additional code optimization for intra-operative planning.

Acknowledgments. Financial support from NIH grant R21 CA120232-01 is gratefully acknowledged. The authors would also like to thank the physicians, therapists and staff at the Vancouver Cancer Center who have contributed in this project. 


\section{References}

1. Noble, J.A., Boukerroui, D.: Ultrasound image segmentation: a survey. IEEE Trans. Med. Imaging 25(8), 987-1010 (2006)

2. Pathak, S.D., Haynor, D.R., Kim, Y.: Edge-guided boundary delineation in prostate ultrasound images. IEEE Trans. Med. Imaging 19(12), 1211-1219 (2000)

3. Boukerroui, D., Baskurt, A., Noble, J., Basset, O.: Segmentation of ultrasound images-multiresolution 2D and 3D algorithm based on global and local statistics. Pattern Recognition Letters 24(4-5), 779-790 (2003)

4. Zaim, A.: An edge-based approach for segmentation of prostate ultrasound images using phase symmetry. In: ISCCSP 2008, pp. 10-13 (2008)

5. Nascimento, J.C., Marques, J.S.: Robust shape tracking with multiple models in ultrasound images. IEEE Trans. Image Process 17(3), 392-406 (2008)

6. Huang, X., Metaxas, D.N.: Metamorphs: deformable shape and appearance models. IEEE Trans. Pattern Anal. Mach. Intell. 30(8), 1444-1459 (2008)

7. Thevenaz, P., Unser, M.: Snakuscules. IEEE Trans. Image Process 17(4), 585-593 (2008)

8. Nanayakkara, N.D., Samarabandu, J., Fenster, A.: Prostate segmentation by feature enhancement using domain knowledge and adaptive region based operations. Phys. Med. Biol. 51(7), 1831-1848 (2006)

9. Hodge, A.C., Fenster, A., Downey, D.B., Ladak, H.M.: Prostate boundary segmentation from ultrasound images using 2D active shape models: optimisation and extension to 3D. Comput. Methods Programs Biomed. 84(2-3), 99-113 (2006)

10. Kachouie, N.N., Fieguth, P., Rahnamayan, S.: An elliptical level set method for automatic TRUS prostate image segmentation. In: Proc. IEEE International Symposium on Signal Processing and Information Technology, pp. 191-196 (2006)

11. Penna, M.A., Dines, K.A., Seip, R., Carlson, R.F., Sanghvi, N.T.: Modeling prostate anatomy from multiple view TRUS images for image-guided HIFU therapy. IEEE Trans. Ultrason Ferroelectr. Freq. Control 54(1), 52-69 (2007)

12. Gong, L., Pathak, S.D., Haynor, D.R., Cho, P.S., Kim, Y.: Parametric shape modeling using deformable superellipses for prostate segmentation. IEEE Trans. Med. Imaging 23(3), 340-349 (2004)

13. Badiei, S., Salcudean, S.E., Varah, J., Morris, W.J.: Prostate segmentation in 2D ultrasound images using image warping and ellipse fitting. Med. Image Comput. Comput. Assist. Interv. Int. Conf. 9(Pt 2), 17-24 (2006)

14. Mahdavi, S., Salcudean, S.E.: 3D prostate segmentation based on ellipsoid fitting, image tapering and warping. In: Conf. Proc. IEEE Eng. Med. Biol. Soc., vol. 1, pp. 2988-2991 (2008)

15. Zhan, Y., Shen, D.: Deformable segmentation of 3-D ultrasound prostate images using statistical texture matching method. IEEE Trans. Med. Imaging 25(3), 256$272(2006)$

16. Tutar, I.B., Pathak, S.D., Gong, L., Cho, P.S., Wallner, K., Kim, Y.: Semiautomatic 3-D prostate segmentation from TRUS images using spherical harmonics. IEEE Trans. Med. Imaging 25(12), 1645-1654 (2006)

17. Abolmaesumi, P., Sirouspour, M.R.: An interacting multiple model probabilistic data association filter for cavity boundary extraction from ultrasound images. IEEE Trans. Med. Imaging 23(6), 772-784 (2004) 